\title{
Making Sense of Non-Individuals in Quantum Mechanics
}

\author{
Jonas R. B. Arenhart, Otávio Bueno, and Décio Krause
}

\begin{abstract}
"It is only a slight exaggeration to say that good physics has at times been spoiled by poor philosophy."

(Heisenberg [1998], p. 211)
\end{abstract}

\section{Motivation}

As the epigraph by Heisenberg suggests, physics and philosophy may both benefit from a beneficial exchange in which one may enlighten the other. Physics can illuminate philosophy, and philosophy can illuminate physics. Of course, some may think that philosophy has nothing to contribute to physics (see Weinberg [1992]), and although we shall not provide a detailed defense of why we take philosophy to be relevant for science in general, we want to defend the relevance of ontology, as a field of metaphysics, to physics and to what physics is about. We stress, in this work, through a case study, the way in which ontology, as a philosophical field, can engage with physics, particularly in clearing the ground for the understanding of the nature of physical reality.

Ontology is concerned with what exists and with what kinds of things exist. Although this description may sound abstract and far from the concerns of physics, the relation between ontology and physics is a close one. Of course, we are not claiming that physics cannot be successful without ontology. If that were the case, ontology would be required for physics, and it is not. However, physicists work with ontological problems all the time. For instance, when it is claimed that, in general relativity, space and time are no longer independent and that a new kind of entity is required, space-time, this is a physical move with significant ontological consequences. It affects directly how the furniture of the world looks like.

Physicists need not be concerned with ontological problems raised by physics, just as one need not be familiar with the Peano axioms in order to be able to use arithmetical operations. But the point is that ontology is part of the enterprise, shared by most physicists, of obtaining information about how the world works and what it is made of. What kinds of things are there? Particles, fields, space, time? What are they like? Answering questions like these is part of the articulation of an understanding of physical reality. As a result, the furniture of the world is involved in such understanding. Ignoring those questions and their importance may prevent one from getting closer to the most fundamental problems.

In this work, we will not focus on such general questions, but rather on a very specific case study: assuming that quantum theories deal with "particles" of some kind (point particles in orthodox non-relativistic quantum mechanics, field excitations in quantum field theories), what kind of entity can such particles be? One possible answer, the one we shall examine here, is that they are not the usual kind of object found in daily life: individuals. Rather, we follow a suggestion by Erwin Schrödinger - among others, as will become clear below - according to which quantum mechanics poses a revolutionary kind of entity: nonindividuals. While physics, as a scientific field, is not concerned with whether entities posited by a specific physical theory are individuals or not, answering this question is part 
of the quest for a better understanding of physical reality. Here lies, in large measure, the relevance of ontology.

\section{Introduction}

There is little doubt that quantum entities are difficult to categorize. Quantum mechanics introduces so many oddities that it is easier to state what quantum entities are not than to affirm what they are. (We use 'entity' here as a term that is neutral regarding whether the things that are referred to have well-defined identity conditions or not.) According to some of the first creators of quantum theory, quantum entities are non-individuals. This view is now known as the Received View on quantum non-individuality (henceforth, for the sake of brevity, "Received View"; see French and Krause [2006], Chapter 3, for further historical details on the view).

In a section aptly called "A particle is not an individual", Schrödinger [1998] advanced one of the formulations of the Received View. One passage is worth quoting in full:

This essay deals with the elementary particle, more particularly with a certain feature that this concept has acquired-or rather lost—in quantum mechanics. I mean this: that the elementary particle is not an individual; it cannot be identified, it lacks "sameness". [...] In technical language it is covered by saying that the particles "obey" a new fangled statistics, either Bose-Einstein or Fermi-Dirac statistics. The implication, far from obvious, is that the unsuspected epithet "this" is not quite properly applicable to, say, an electron, except with caution, in a restricted sense, and sometimes not at all. (Schrödinger [1998], p. 197)

Several significant points are made in this passage. It is noted that quantum particles (i) are not individuals, (ii) cannot be identified, (iii) lack "sameness", and (iv) cannot be referred to by the use of "this", at least not typically. Of course, it is not clear, by considering this quotation alone, what Schrödinger's conception of identification, individuality, and sameness ultimately is, nor is it specified what the proper relations among these concepts are. But a central feature of his view becomes salient in another important passage. He notes:

I beg to emphasize this and I beg you to believe it: it is not a question of our being able to ascertain the identity in some instances and not being able to do so in others. It is beyond doubt that the question of "sameness", of identity, really and truly has no meaning. (Schrödinger [1996], pp. 121-122)

Here, it is emphasized that the very question of the identity of quantum entities, the question of their "sameness", has no meaning. As a result, the difference between these entities, provided their sameness is meaningless, has no meaning either. One still need to examine, of course, what exactly is the relation between the lack of sameness (or identity) of quantum entities, on the one hand, and their lack of individuality, on the other. It seems that Schrödinger takes them all to be conceptually the same: to "lose" one's individuality just is to lose one's identity. On his view, the question of the identity of quantum particles in general makes no sense. The proper understanding of the relations between these concepts, and the kind of view that results from them in the context of quantum particles, is the topic of this paper.

These issues were also central to another contributor to the development of quantum theory. In a classical passage, in which the issues of identity and individuality were prominent, Hermann Weyl points out that:

[...] the possibility that one of the identical twins Mike and Ike is in the quantum state $E_{1}$ and the other in the quantum state $E_{2}$ does not include two differentiable cases which are 
permuted on permuting Mike and Ike; it is impossible for either of these individuals to retain his identity so that one of them will always be able to say 'I'm Mike' and the other 'I'm Ike'. Even in principle one cannot demand an alibi of an electron! (Weyl [1950], p. 241)

The questions of discernibility and of an "alibi" of a quantum particle is clearly posed. Once quantum particles, such as electrons, are in an entangled state, it cannot be determined which particle is in which state. In other words, it cannot be settled which particle is which. There is nothing - no property, no special ingredient - that could act as an alibi to discern electrons. In this respect, it is their indiscernibility rather than their identity that should take center stage. Differently from what Schrödinger suggests, perhaps identity need not lose its meaning, provided that indiscernible things can still be numerically distinct (or identical). As will becomes clear, to articulate this proposal it is required that identity and indiscernibility be distinguished. In classical logic and standard mathematics, identity is formulated in terms of indiscernibility. So, in order to keep one and change the other, one needs to resist this identification and clearly separate the two notions. (We will return to this below.)

As these quotations illustrate, when it comes to the investigation of the nature of quantum entities, various possibilities are open. One can examine the commonalities between the conceptions underlying Schrödinger's and Weyl's approaches or pursue their differences. A major feature that is common to both is that they seem to suggest that something is lost by quantum entities: something that marks a difference between quantum entities and classical entities.

In this paper, we address the articulation of the Received View and the conception of nonindividuality that it attributes to quantum entities. As we discuss in Section 2, the conception can be formulated in distinct ways, some more radical, others more conservative, at least with regard to the role of the concept of identity as used in quantum theories. The main issue turns on the behavior of identity and its relation with individuality. Central to the Received View is the claim that identity makes no sense, a claim that, as just noted, Schrödinger seems to have favored. We discuss, in Section 3, how to make metaphysical sense of that idea. The bare claim that identity makes no sense should be accompanied by an account of how this view entails that particles are not individuals. In Section 4, we discuss the formal consequences of the idea, and apply the Received View to suggest a revision of classical logic. In Section 5, we draw some consequences of this case study to the significance of research in the foundations of physics.

\section{The Received View}

Common to the claims of both Schrödinger and Weyl quoted above is an important point: what is responsible for the strange metaphysical behavior of quantum particles is the statistics they obey. Behind this trait one finds encapsulated the so-called permutation symmetry (PS). According to PS, quantum states should be symmetric (or anti-symmetric) with regard to the permutation of labels of particles. As a result, if we are to represent a system composed by two particles $x_{1}$ and $x_{2}$, so that one of the particles is in a region $A$ and the other is in a region $B$, it cannot be determined which particle is in which region. (A qualification is in order: assuming that the underlying "space" is Newtonian and thus, mathematically, its topology is Hausdorff, it follows that two separate points can always be discerned by disjoint open balls centered on the points; see Krause [forthcoming]. Attention to the interface between the mathematical framework and the physical setup is important.) In this case, non-symmetric wave functions, $\Psi_{A}\left(x_{1}\right) \Psi_{B}\left(\mathrm{x}_{2}\right)$ or $\Psi_{A}\left(x_{2}\right) \Psi_{B}\left(x_{1}\right)$, are unable to describe the situation alone; what is needed is a superposition of both of them: 


$$
\Psi_{A B}=\Psi_{A}\left(x_{1}\right) \Psi_{B}\left(x_{2}\right) \pm \Psi_{A}\left(x_{2}\right) \Psi_{B}\left(x_{1}\right), \text { except for a normalization factor. }
$$

Thus, the permutation of $A$ with $B$ results either in the same state $\Psi_{A B}$ in the case of bosons or in the state $-\Psi_{A B}$ in the fermions' case. (See French and Krause [2006], Chapter 4, for a discussion of the physical aspects of this situation and an examination of how far the metaphysics can go.) More importantly, the square of the resulting wave function, which gives us the relevant probabilities, is preserved (since $\left|\Psi_{A B}\right|^{2}=\left|-\Psi_{A B}\right|^{2}$ ). Hence, if $A$ stands for an arbitrary observer and $P$ is a permutation operator, then

$$
\left\langle\psi_{A B}|A| \psi_{A B}\right\rangle=\left\langle P \psi_{A B}|A| P \psi_{A B}\right\rangle .
$$

As it turns out, this is as far as one can go based on the quotes given above. Any additional steps will break the shared agreement, given that different metaphysical conclusions will be made in light of the same physical fact.

In both of his claims above, Schrödinger seems to identify "sameness" and identity, so that the fact that one cannot attribute sameness to the particles also means that one cannot attribute identity to them. Individuality is lost as a result of the lack of sameness. Given that it makes no sense to state that one particle is the same as the other and given that it is not possible refer to a particle as "this" one, particles are no longer individuals.

Of course, the issue is more complex than these considerations suggest. If it were possible to determine that there is one particle and then another, it would certainly make sense to state that they are different. But this is not quite what Schrödinger claims. At this point, an additional ingredient should be added to make clear what Schrödinger's conception of individuality ultimately is. With regard the typical principle of individuality of the metaphysicians, which accounts for what an entity is in contrast to others, Schrödinger advances a particular space-time principle of individuation, one which accounts for the individuality of an item in terms of its spatiotemporal position (see French and Krause [2006], Chapter 1, for details).

In discussing the individuality of familiar objects, Schrödinger ([1998], p. 204) claims that science has taken for granted the permanence of pieces of matter, and this is what accounts for the identity and individuality of objects. This is manifested in one's confidence when the identity of familiar objects becomes an issue:

When a familiar object reenters our ken, it is usually recognized as a continuation of previous appearances, as being the same thing. The relative permanence of individual pieces of matter is the most momentous feature of both everyday life and scientific experience. If a familiar article, say an earthenware jug, disappears from your room, you are quite sure somebody must have taken it away. If after a time it reappears, you may doubt whether it really is the same one-breakable objects in such circumstances are often not. You may not be able to decide the issue, but you will have no doubt that the doubtful sameness has an indisputable meaning - that there is an unambiguous answer to your query. (Schrödinger [1998], p. 204)

Compare the view articulated in this passage with the one Schrödinger advanced earlier when he claimed that the notion of identity makes no sense for quantum entities (see the quotation from Schrödinger [1996], pp. 121-122, in the previous section). While ordinary objects typically are supposed to have well-defined identity conditions, which allows one to answer questions about their identity over time (even if, in some cases, one may be unable to decide the issue), for quantum objects such questions do not even make sense. As a result, there is simply no fact of the matter regarding the individuality (as well as the 
identity or sameness) of quantum particles. In fact, in the case of quantum particles, situations involving distinct observations of an object through time generate problems that prevent the individuality of the items in question from making sense. As Schrödinger notes:

Even if you observe a similar particle a very short time later at a spot very near to the first, and even if you have every reason to assume a causal connection between the first and the second observation, there is no true, unambiguous meaning in the assertion that it is the same particle you have observed in the two cases. The circumstances may be such that they render it highly convenient and desirable to express oneself so, but it is only an abbreviation of speech; for there are other cases where the "sameness" becomes entirely meaningless [...]. (Schrödinger [1996], p. 121)

Schrödinger highlights the need for identity in order to claim that an entity is observed in distinct places at distinct times. It is then just one step to add that without the possibility that a particle observed at one instant of time $t_{1}$ is the same as a particle observed at a later time $t_{2}$, individuality is lost. Given that, on this view, it makes no sense to state that those particles are the same (or different), identity loses its meaning. As we have already noted, identity, individuality, and sameness are taken as conceptually the same by Schrödinger.

Based on these considerations, a straightforward version of the Received View emerges. Quantum particles are not individuals given that they have no well-determined trajectories in space-time, and it is not possible to identify distinct detections of an entity as being detections of the same entity. (We will return to this view in the next section and will provide additional details there.)

However, this is not the only way to articulate the Received View via space-time continuity. Another form is to keep the restriction that quantum entities fail to have welldefined spatiotemporal trajectories (as is the case in most versions of the theory), and thus insist that these entities are non-individuals in this sense, but not connect this lack of individuality with a lack of identity. That is, one could keep a form of space-time individuality, but separate individuality from the logical relation of identity (see the suggestion in French and Krause [2006], p. 153, and Arenhart [2017]). Perhaps Schrödinger could be interpreted as suggesting this view: identity does not apply to quantum particles, but this is no violation of the Principle of Identity of Indiscernibles (PII), according to which some quality always discerns numerically distinct items. Since, on this view, PII is supposed to apply only to individuals, which quantum particles are not, such particles do not provide a violation of the principle. Along these lines, another version of the Received View could be advanced, along with a distinc conception of nonindividual.

But another option, which is less revisionary regarding the role of identity than Schrödinger's, can still be pursued. One can take literally the claim that quantum particles are non-individuals but resist to follow Schrödinger in making the further claim that identity, as a logical relation, loses its meaning. In fact, if Weyl's "alibi" is taken to refer to a principle of individuality, then it makes sense to claim that even though no alibi is available for quantum particles, this lack of alibi need not be connected with the lack of meaning of identity (hence, identity would be metaphysically deflated, as recommended by Bueno [2014]). In order to do that, it is enough that one resists binding so tightly an item's individuality with its identity. Let us explore this option further, given that pursuing this route provides an additional (alternative) version of the Received View. (See also Arenhart [2017] for a discussion of alternative formulations of the Received View, which do not involve abandoning identity.) 
One can take the alibi Weyl refers to as involving a property that distinguishes a particle bearing it from any other particle. It is always possible to differentiate a classical particle (that is, a particle described by classical mechanics) from another particle by at least one property. Of course, it cannot be a state-independent property (given that particles of the same kind share such properties), but at least their spatiotemporal location distinguishes them. On this view, no two classical particles occupy the same location at the same time, due to a principle of impenetrability. This means that numerically distinct particles have their difference grounded in a property that accounts for their numerical diversity and their individuality.

This trait leads to the validity of PII in classical mechanics. According to this principle, as noted above, numerically distinct particles are always discernible by some quality. Entities are individuals due to the fact that once two of them are present, there is always some property that accounts for their numerical difference: this is their alibi. In light of PII, an alibi is always available in classical mechanics. As a result, classical particles are indeed individuals.

In contrast, quantum particles have no alibi, nothing that accounts for their individuality. Not even spatiotemporal location can be employed to this effect. Due to the permutation symmetry, quantum particles are indiscernible by their properties, including both state dependent and state independent ones. Hence, the version of PII presented earlier, according to which there is always some property that accounts for the numerical diversity of particles, fails in quantum mechanics. The result is clear: as Weyl noted, there is no alibi for quantum entities (see French and Krause [2006], Chapter 4, for further discussion).

It could be argued that if properties are unable to account for the numerical difference of quantum particles, perhaps some relations could do that, such as the relation "to have spin opposite to" in a given spatial direction. But this proposal is still unable to account for the particles' individuality. After all, if $x$ has spin opposite to $y, y$ also has spin opposite to $x$ (the relation is symmetric). While no particle has spin opposite to itself (the relation is irreflexive), there is no quantum mechanical fact of the matter to determine which of $x$ or $y$ has spin up in a given direction, and which has spin down in that same direction. Thus, those relations, called weakly discernible relations, in principle can account for the numerical diversity of the particles (although whether they do account for that is still debatable; see French and Krause [2006], Chapter 4). Despite that, they are unable to provide an alibi for the particles in question since weakly discernible relations are unable to individuate such particles. Accounting for the particles' numerical diversity (if at all) is the closest one can get in quantum mechanics to discernibility (see Muller and Saunders [2008], and the discussion in Lowe [2016]).

However, if weakly discernible relations are implemented in a mathematical context whose underlying set theory is ZFC (Zermelo-Fraenkel set theory with the axiom of choice), as is the case of Muller and Saunders [2008], all entities become fully discernible and identifiable in virtue of the resources of set theory alone. (We return to this point and provide the argument below.) Thus, there is a tension between the motivation for the introduction of weakly discernible relations and the adopted set-theoretic framework.

In principle, if the option of maintaining that identity holds for quantum particles can be fully worked out, one could claim that they are different or identical, without thereby implying that they are individuals. What is required, as we have been suggesting, is that their individuality be grounded in some kind of alibi (in Weyl's sense) that is not formulated in terms of identity. 
There are additional possibilities to articulate alibis (that is, principles of individuality) without requiring the removal of identity (see Arenhart [2017]). It is enough that the content of identity be deflated from the metaphysical content that would be required if identity also played the role of a principle of individuality. (For a defense that identity should be deflated, see again Bueno [2014].) As will become clear below (when a formal approach to identity is discussed), identity can be thought of as something very minimal, and without much metaphysical content, just in terms of two features: reflexivity (that is, every object is identical to itself) and substitutivity (if $x$ is identical to $y$, then if $x$ is $F$, so is $y$ ). One could add some metaphysical content to identity, so that it can be used as a principle of individuality. But that changes identity by making it more substantive than it needs to be. Schrödinger, of course, does not seem to follow this path since he appears to keep identity and individuality very closely connected. As a result, insisting on nonindividuality requires abandoning identity, at least for quantum entities. In what follows, we investigate the prospects for a Schrödingerian approach to non-individuality.

\section{Making Sense of Losing Identity}

If one is to pursue the option that seems to be suggested by Schrödinger-namely, that individuality and identity go together, and that one cannot have non-individuals without abandoning also the relation of identity - it is crucial to explain in detail why identity should go hand in hand with individuality regarding quantum particles.

One of the possible ways of doing that consists in exploring the relation between identity and individuality through the notion of haecceity, as it is done, for instance, in French and Krause [2006]. Basically, a haecceity is a non-qualitative property uniquely instantiated by an object, something like an individual essence possessed by a single individual. Each individual has its own essence, which, of course, accounts for its individuality (see further discussion in Lowe [2003]).

Being non-qualitative, a haecceity does not count as a quality able to discern among two individuals. That is, two individuals may share every qualitative property, but still not be the same individual, due to the fact that they have distinct haecceities. As a result, PII (restricted to qualitative properties) may fail and, despite that, individuality is still saved in light of a haecceity. In this sense, having a haecceity is what French and Krause ([2006], Chapter 1) call a "Transcendental Individuality" principle: that which confers individuality over and above an item's qualities.

This point has an important formal counterpart. If the underlying mathematical framework in which quantum theories are formulated is that of ZFC, one should consider, in particular, the resulting models - the structures in which quantum mechanics holds. Within these structures, it is possible that certain entities cannot be discerned based on the resources of quantum theories alone. However, outside such structures, it is possible to discern the entities in question, and this can be thought as a formal expression of "transcendental individuality". (Clearly, the relevant structures cannot be rigid.)

The problem, however, is not to account for an item's individuality, but rather for its nonindividuality. How can haecceity achieve that? The answer seems to be: through the notion of identity. As French and Krause put it:

[...] the idea is apparently simple: regarded in haecceistic terms, "Transcendental Individuality" can be understood as the identity of an object with itself; that is, ' $a=a$ '. We shall then defend the claim that the notion of non-individuality can be captured in the quantum context by formal systems in which self-identity is not always well-defined, so 
that the reflexive law of identity, namely, $\forall x(x=x)$, is not valid in general. (French and Krause [2006], pp. 13-14)

That is, a haecceity may be formally represented by self-identity. Plato's individuality, if it were attributed by a haecceity, would consist in his bearing the property of being identical with Plato. This, of course, is a non-qualitative property, and it is able to connect identity (as a logical concept) and individuality (as a metaphysical concept). The quote above also provides the basic idea to make sense of non-individuality within a framework that takes into account the Schrödingerian claims that quantum entities are not individuals and that identity makes no sense for them. In order to accommodate metaphysically the idea that identity has no meaning for quantum particles, it is enough that the reflexive law of identity fails or does not hold in general. Thus, not everything is self-identical. In light of this connection between self-identity and haecceity, those entities for which the law fails are non-individuals: they lack a haecceity, which is the individuation principle.

French and Krause acknowledge explicitly that the connection between identity and individuality is particularly tight:

We are supposing a strong relationship between individuality and identity [...] for we have characterized 'non-individuals' as those entities for which the relation of self-identity $a=a$ does not make sense. (French and Krause [2006], p. 248)

This is only one of the possible ways to accommodate metaphysically the combination of non-individuality and the loss of identity. This proposal allows one to make a good case for the failure of identity, given that the relation between individuality and identity is very clearly established in this approach. However, in addition to burdening identity with the role of attributing individuality, there is another disadvantage of adopting this approach to non-individuality: it takes us very far from the Schrödingerian ideas we started with. Of course, it allows us to make sense of the claim that identity and difference do not apply to quantum entities. But the lack of haecceity arguably was not what Schrödinger had in mind in his discussion of identity and identification of quantum particles. Rather, as discussed above, he seems to favor an account of individuality framed explicitly in terms of spatiotemporal trajectories.

The following two conditions seem to articulate better the conception of an entity being individuated by its spatiotemporal position, in the sense that an entity satisfying these two conditions should be counted as an individual (see also Bueno [2014] and Bueno [forthcoming]):

(A) Identity conditions: an individual has (clearly determined) identity conditions.

(B) Persistence conditions: an individual persists over time (despite changes).

Note that these minimal conditions are satisfied by what is typically considered individuals (such as, chairs, cherries, or chariots). In particular, as Schrödinger emphasizes, an earthenware jug would be an individual according to this approach, and so are classical particles, given that their well-determined trajectories grounds both their persistence and their identity conditions (see French and Krause [2006], Chapter 2). Quantum particles in Bohmian mechanics are also individuals according to this characterization: they have trajectories attributed by hidden variables.

It should be noted that there are at least two ways of satisfying persistence condition (B):

(B.1) Essential traits: as long as certain essential traits (or necessary properties) of an individual are preserved, the individual remains in existence. 
(B.2) Closest continuers: given an individual $i$ that satisfy condition (A), at each moment of time the closest continuer individual to $i$ (the one that shares most properties with $i$ ) is taken to be $i$ (Nozick [1981], Chapter 1).

Of course, a haecceity could be an essential trait, and in this way, haecceities could be used to account for the permanence of an individual. Given that we have already suggested avoiding a theory of haecceities to account for individuality and to frame an approach to non-individuality, we favor the less metaphysically committing option (B.2). The idea is that an individual persists through a sequence of closest continuers, which, taken together, account for the permanence of an individual over time despite the changes it undergoes.

Given this theory of individuality (formulated by the conjunction of conditions (A) and (B.2)), for something to be a non-individual, three options emerge: condition (A) can be violated; condition (B.2) can be undermined, or both conditions can fail. Quantum entities, as the discussion of Schrödinger's view indicates, violate both conditions. This is a Humean point: there appears to be no causal connection that would allow one to determine that similar objects detected in different moments in time are, in fact, the same. In the quantum case, consider some quantum entities that have no continuous trajectory. One cannot look for a quantum mechanical justification to connect two observations of two such entities through a single trajectory. Nothing in the theory allows us to do that (unless one is a Bohmian). As a result, as we have seen, Schrödinger claimed that identity makes no sense for those entities, given that there is no fact of the matter to determine whether the two observations correspond to the same entity or not. The question of the identity of the observed entities ends up being entire ungrounded.

This accounts for both the non-individuality of the particles and the fact that identity does not apply to them. This metaphysical picture is closer to what Schrödinger had in mind, it seems, and it is less inflated than the one first suggested by French and Krause [2006], which proceed through the concept of a haecceity. However, both approaches require a corresponding rejection of the overall validity of identity. One of the ways to accommodate such a limitation of identity is through logics that restrict identity, the so-called "nonreflexive logics". We turn to them now.

\section{The Formal Approach to Identity}

It is important to be clear about what identity is, particularly when it is stated that quantum objects lack identity. Throughout this work, we have been using the term "identity" in the sense of what is typically called standard identity (or simply "identity", for short) as conceptualized by classical logic and standard mathematics.

But there is a pre-theoretical conception of identity (let us call it a numerical identity, for lack of a better word). This conception states that every object is identical just to itself and to nothing else. According to this informal view, when it is coupled with the tight connection between identity and individuality just discussed, it follows from the fact that all objects have identity that they are individuals. So, under this interpretation, the informal notion of identity is closely related to that of individuality.

The informal view of identity discussed above is generally thought to be encapsulated in classical logic, or in other systems of logic, which share the basic features of identity. Let us focus on classical logic, for the sake of brevity. Classical logic takes identity as a binary relation between objects of the domain. Identity statements are usually written as $a=b$, (or as $a \neq b$, depending on the case), in order to express that the objects denoted by $a$ and by $b$ are the same, they are identical (or are not the same, they are different). This intuitively means that there are no two objects, but just one, which can be named 
(described) by such expressions. A typical case is the famous identity statement "Hesperus = Phosphorus" (Frege [1960]). True statements such as this make reference to the identity of the objects of the domain, and 'Hesperus' and 'Phosphorus' denote the same object. One should consider both the syntactic characterization of the notion of identity (given by the binary predicate ' $=$ ') and the semantic characterization in which the identity of the objects of the domain of interpretation is at issue (we denote the domain by $D$ ).

Let us consider the semantics first. The identity of $D$ is taken to be the set $I_{D}=\{\langle x, x\rangle: x \in$ $D$ \}. This clearly presupposes that the identity of the elements of $D$ is well determined, and the metamathematical framework is consistent with this fact. If one assumes a standard semantics, that is, a semantics built in a standard set theory such as ZFC, then this assumption is met, given that the identity of all sets is presupposed. (We will return to this point below.) $I_{D}$ is a set. According to Cantor, it is thus "a collection of definite and distinct objects of our intuition or of our thought" (Cantor [1955], p. 85). This informal, circular idea of a set (of course, it is not a definition), which accounts for sets in terms of collections, is couched in terms of numerical identity.

The problem concerns the syntactic side. Is it possible to axiomatize a first-order logic having a primitive binary predicate for identity having the set $I_{D}$ as its sole interpretation? That is, is it possible to provide a definition (or a group of postulates) such that the identity predicate has just $I_{D}$ as a model? The answer, we argue, is negative. Let us see why.

To begin with, it is important not to confuse numerical identity with the notion of identity in standard logic and mathematics. Arguably, it is primarily the latter that can be rigorously dealt with. Suppose that the language $L$ under consideration is first-order. Two cases emerge. First, $L$ contains just a finite set of primitive predicates. In this case, we can "define" identity by exhausting all predicates. An example suffices: suppose that the predicates are two monadic predicates $P$ and $Q$ and a binary predicate $R$. Thus, $a=b$ can be "defined" as follows:

$$
\text { (1) } a=b \leftrightarrow(P a \leftrightarrow P b) \wedge(Q a \leftrightarrow Q b) \wedge \forall x((R x a \leftrightarrow R x b) \wedge(R a x \leftrightarrow R b x))
$$

The problem with this "definition" is that clearly there can be additional predicates not belonging to the language that could distinguish $a$ and $b$, not to mention the possibility of there being some kind of haecceity that achieves that (as we noted in the beginning of the previous section when we made the point about the "formal counterpart" regarding haecceity). In fact, (1) stands only for the indiscernibility of $a$ and $b$ regarding the predicates of $L$.

Second, usually first order languages introduce identity as a primitive binary predicate ' $=$ '. In this case, the standard formulation makes use of two postulates, namely:

(R) Reflexivity: $\forall x x=x$

(S) Substitutivity: $x=y \rightarrow(\alpha x \rightarrow \alpha[y / x])$, where $x$ and $y$ are individual variables, $\alpha x$ is a formula having $x$ free, and $\alpha[y / x]$ results from the substitution of $y$ for $x$ in some free occurrences of $x$, in which $x$ and $y$ are distinct variables.

(Note the use of identity in the very formulation of the substitutivity rule: the variables $x$ and $y$ need to be distinct, that is, not identical.) From these postulates, it follows that identity is symmetric and transitive. Thus, it is an equivalence relation as well as a congruence relation due to the presence of substitutivity. Logicians say that identity is the finest congruence over the domain in the sense that if $\cong$ is another congruence, then $a=b$ entails $a \cong b$, for all $a$ and $b$. 
But things are not so easy. Postulates (R) and (S) cannot guarantee that the interpretation of the predicate ' $=$ ' is the set $I_{D}$. In fact, it can be shown that a congruence, other than identity, can be defined over the domain that also models the predicate of identity (da Costa and Bueno [2009]; Krause and Arenhart [2018]). In other words, from the point of view of $L$, it cannot be known whether one is working with a structure where ' $=$ ' is interpreted as the identity of the domain $D$, namely, the set $I_{D}$, or in terms of another structure that has the defined congruence as the interpretation of syntactic identity. These structures are elementary equivalent.

Leaving first-order languages behind, higher-order languages should then be considered. It suffices to consider $L$ as a second-order language (the generalization to other higherorder languages is immediate). In this case, identity can be (allegedly) "defined" in terms of indiscernibility (indistinguishability) by what is called Leibniz Law, namely:

$$
\text { (2) } x=y \text { if, and only if, } \forall F(F x \leftrightarrow F y) \text {, }
$$

where $x$ and $y$ are variables for individuals and $F$ is a variable for properties of individuals. The right-side of the biconditional expresses the indiscernibility of $x$ and $y$, and it states that the objects that stand for $x$ and $y$ have the same properties (hence they also share all relations).

The problem now is with the semantics. Suppose that the domain is the non-empty set $D=$ $\{1,2,3,4,5\}$ and that our second-order language has three monadic predicate constants $-P, Q, R$ - and two individual constants $-a$ and $b$. Consider the following interpretation: 1 is assigned to $a$ and 2 to $b$. Furthermore, the extensions of the predicates are interpreted as the following sets: $A=\{1,2,3\}, B=\{1,2,4\}$, and $C=\{1,2,5\}$. Thus, since 1 and 2 belong to all sets, it follows that $a$ and $b$ have all properties in common. In other words, the right-side of (2) holds, despite the fact that $1 \neq 2$.

The only way of guaranteeing that (2) will have its full intuitive meaning is to add to the semantics all subsets of $D$, that is, to consider what Church calls principal interpretations (Church [1956], p. 307). But then, as is well known, completeness is lost.

As these considerations make clear, identity is not a simple concept when one tries to provide a rigorous account of the intuitive idea. But from a logical point of view, this is what classical logic presents us with. Based on this theory of identity, which is called classical theory of identity (CTI), we can consider stronger systems, such as various set theories.

As is well known, there are several non-equivalent set theories with distinct properties and which yield significantly different, and even incompatible, theorems. For instance, ZFC includes the axiom of choice; Quine-Rosser's NF system does not: it is incompatible with this axiom (Forster [2014]). In ZFC, if consistent, there is no Russell set, namely, $R=\{x: x \notin$ $x\}$, but in some paraconsistent set theories, this set is legitimate (da Costa, Krause and Bueno [2007]). It can be proved, in ZFC, that there are sets that are not Lebesgue measurable, but in "Solovay set theory" all sets are Lebesgue measurable (Maitland Wright [1973]). What is remarkable is that all these set theories invoke the same theory of identity, namely, CTI. Thus, our considerations apply to all of them.

It is undeniable that set theory is the most widely used basis for standard mathematics, that is, the part of mathematics that can be developed in theories such as ZFC. This is also the mathematics that underlies quantum theories. In fact, it is unclear what kind of quantum mechanics could be developed in a system such as NF, given its incompatibility 
with the axiom of choice (AC). After all, AC is necessary for the usual mathematical formulation of quantum mechanics, so that it can be guaranteed, for instance, that the relevant Hilbert spaces have a basis. (Of course, quantum mechanics can be developed in many different ways that need not rely on von Neumann's approach; see Styer et al. [2002].)

It is a remarkable fact, we noted, that in all of these set-theoretic frameworks, all objects are individuals, in the sense that all of them have identity. In other words, given any objects (that is, any sets; the case of Urelemente will be mentioned soon), there is always a way to distinguish them, if not effectively, at least in principle. The proof is immediate. Given a certain object $a$, which is either a set or a Urelement, the postulates of a set theory enable us to form the set $\{a\}$, the singleton of $a$. (As is well known, there are pure set theories, containing only sets, and impure set theories, systems that also include atoms - the Urelemente in the original Cantor's terminology. These atoms are not sets but can be elements of sets.) Define the "property" $I d_{a}(x)=: x \in\{a\}$. The only object that has such a property is $a$ itself, so $a$ has at least one property distinguishing it from any other object. Leibniz law applies and, thus, there cannot be indistinguishable but non-identical objects.

Indiscernible entities can be accommodated in a set theory via equivalence relations. The elements of an equivalent class can be taken as representing the same object, but this is clearly a mathematical trick and does not work as part of a philosophically well-motivated proposal. A trick similar to this is used in orthodox quantum mechanics when symmetric and anti-symmetric wave-functions are chosen to stand for certain quantum systems: functions are selected that do not alter the probabilities when particle labels are exchanged. (This trick was called "Weyl's strategy" since it was used by Hermann Weyl; see French and Krause [2006], §6.5.1.)

As a result, within standard mathematics, there are no absolutely indiscernible objects as quantum objects are said to be in certain situations. Thus, if we use standard mathematics in our preferred formulation of quantum mechanics (the same point applies to quantum field theories), from the simple fact that there are two quantum objects, it results from the mathematics alone that the objects are different (they are not identical), and by Leibniz law, there is at least one property that one of them has and the other does not. However, if the objects in question are indiscernible, such as two bosons in the same state, which property would that be? The assumption of the existence of such a property amounts to the introduction of hidden variables - even in those formulations of quantum mechanics that do not accept them. But that there is such a property follows from Leibniz law (which, as noted, is part of the package formed by classical mathematics, which includes a corresponding logic, and the standard theory of identity). Thus, in any situation, given two quantum objects, there is a difference between them. Such a difference cannot be given by a substratum (a haecceity) since the existence of such a substratum is ruled out in quantum theories (see Teller [1998]). The difference can be expressed in terms of a bundle theory of properties, which leads to the conclusion that there is a property that only one of the quantum objects in question have, but not the other. The problems is that according to quantum theories, assuming their usual interpretations, this is not a viable possibility. Otherwise, quantum objects would be discernible. In the end, what is needed is a framework that does not preclude the possibility of indiscernible but potentially distinct systems of entities, a framework that makes room for non-individuals.

An appropriate, philosophically well-motivated, strategy would then be to leave standard set theories behind and adopt a set theory in which identity is not taken to hold in general, namely, a quasi-set theory. This is a mathematical framework which can be used as a metamathematics for quantum theories (see French and Krause [2006], Domenech et al. 
[2008], and Krause and Arenhart [forthcoming]). In this theory, collections (called quasisets) can be formed by absolutely indiscernible elements without thereby becoming identical. As a result, Leibniz law is violated for some objects (although it remains valid for another kind of objects, called classical). These collections of indiscernible entities can have a cardinal, called its quasi-cardinal, even if they do not have an ordinal. The theory provides a framework to examine collections of objects without ordering them, without identifying or individuating them. And differently from classical set theories, the theory offers a framework in which non-individuals can be formulated and thoroughly studied without the incoherence found in the use of classical set theories for the formulation of the foundations of quantum mechanics. (For details, see French and Krause [2006], and Krause and Arenhart [forthcoming].) We conclude this work by noting the significance of foundational studies of physics, of which quasi-set theories provide a clear case.

\section{Conclusion}

In this paper, the metaphysical underpinnings of the idea that quantum entities are nonindividuals have been examined. Schrödinger's claim that identity does not make sense for quantum entities was interpreted, and the connections between this claim and some issues related to continuous trajectories in quantum theory were investigated. The resulting metaphysics of non-individuals assumes a tight connection between identity and individuality, so that if individuality has to go, so does identity. Given that identity is a logical relation, which is part of classical mathematics, and since almost every system of logic has a version with identity, it is important to provide an account of what it is like for a system of logic to have no identity. A few more words on the importance of developing such formalisms and their relation with physics are, thus, in order.

First, consider some reasons to look for alternative mathematics (and logic) for quantum mechanics. Leaving aside historical considerations (an up-to-date analysis, which also considers some historical facts, can be found in Maudlin [2018]), the motivation has to do with the foundations of physics. One could argue that physics works fine with standard (Leibnizian) mathematics (and logic), as it can be seen by considering any book on quantum mechanics. In particular, the argument goes, questions about the foundations of physics could be regarded as "mere philosophical" problems that, on their own, contribute nothing to the clear understanding of physics. That this view is untenable becomes clear by considering some of the papers in Cao [1999], and the significant insights that a careful reflection on the foundations of physics provides. (For the sake of brevity, we will not revisit the various arguments here.)

It could be argued that something similar happens with current physical theories. Current physics works fine with two incompatible theories, namely, the Standard Model of particle physics and general relativity. The former provides the best way developed so far to account for the physics of the small, while the latter offers the best physics of the big, as it were. One or the other is applied depending on the subject matter under study. However, these two theories are logically incompatible with one another, for gravity has not been quantized. Should the situation be left at that, with everyone being encouraged to accept that physics has reached its final limit and no unification is ultimately necessary?

Of course not. Novelties have always emerged when foundational issues have been pursued. In mathematics, this is undeniably the case, as the development of logic and set theory clearly illustrates. There is no reason to think that quantum mechanics would be any different. Indeed, the relevance of string theories, loop gravity, and any other attempt to find a more fundamental theory, in particular the quantization of gravity, cannot be appreciated without acknowledging the significance of foundational research. In fact, 
without the latter, it would be difficult to make sense of why physicists systematically pursue such enterprises.

To look for more suitable mathematical bases for a coherent metaphysical conception of quantum objects as non-individuals is reasonable and even necessary (to prevent inconsistencies). Arguably, no one seems to know, and perhaps no one will ever know, what quantum entities ultimately are. All one has are one's theories. Even the concept of particle changes from theory to theory (see Falkenburg [2007], Chapter 6). Foundational research provides some perspective and insight to pursue the search for understanding that is integral to the attempt of making sense of these issues as well as their significance.

This brings the second topic to be addressed here: ontology. Physicists, in general, have a broad and intuitive idea of what ontology is, but some of them do not find it relevant to their work in physics. Ontology, it was noted, is traditionally occupied with what there is (in the world), with existential questions, such as: "Are there winged horses?", "Are there electrons?", "Are there transcendental numbers?". Metaphysics is more general and includes ontology as a proper part. For instance, Democritus' claim that the world is composed by atoms (indivisibles) is a metaphysical view. It concerns the basic structures of the world. Even in classical logic one finds metaphysical assumptions. For example, classical propositional logic assumes a metaphysical, semantic principle to the effect that the truth of a complex formula depends on the truth of its component formulas, usually referred to as Frege's Principle of Compositionality (Szabó [2017]).

Physics is not different and it also has its share of metaphysical claims. One of them, crucial to the entire discussion examined in this work, is that quantum objects - whether they are particles in orthodox quantum mechanics or field excitations in quantum field theories - are ultimately non-individuals. This claim, of course, does not force us to assume that non-individuals exist. Situations can be presented in a conditional form: if there are things like quantum entities, then they can be interpreted as non-individuals. This process of interpretation, itself an integral part of foundational research, provides a possible approach to the understanding of the nature of such entities.

Just to be clear, we are not asserting that quantum objects are non-individuals. It is unclear how this claim could be established. Rather, the goal is to develop the view as coherently as possible and indicate how it helps one understand quantum entities. Bohmian mechanics (BM) works with a 'classical' metaphysics involving 'classical' individuals, each one having their own identity. But Bohmians should also be careful and acknowledge that their hypothesis is just a hypothesis, a metaphysical view among several others. The physics works fine with BM, at least at the level of its non-relativistic counterpart (we make no commitment regarding relativistic BM). Clearly, Bohmian mechanics provides an additional example of the significance of foundational research.

To conclude, placed in classical metaphysics and in standard underlying mathematics (based on ZFC), quantum objects cannot be completely indiscernible. The resulting theory can provide predictions of quantum phenomena, such as in the two-slit experiment (see Holland [2010]), but these are just predictions able to make the physics work. The problem, however, is the logic that is being invoked is inconsistent with the indiscernibility of the phenomena in question. Quantum statistics, Gibbs paradox, and many other quantum phenomena presuppose absolute indiscernibility. As Wiczek and Devine have said, "in the microworld, we need uniformity of a strong kind: complete indistinguishability" (Wilczek and Devine [1987], p. 135). So, from a logical point of view, predictions are not enough: one need a proper foundational mathematical framework. The commitment that classical logic and standard mathematics have to Leibniz law questions 
their adequacy to accommodate, in a proper way, truly indistinguishable things. A different path is then called for.

\section{References}

Arenhart, J.R.B. [2017]: "The Received View on Quantum Non-Individuality: Formal and Metaphysical Analysis", Synthese 194: 1323-1347.

Bueno, 0. [2014]: "Why Identity is Fundamental”, American Philosophical Quarterly 51(4): 325-332.

Bueno, 0. [forthcoming]: "Weyl, Identity, Indistinguishability, Realism", in Cordero, A. (ed.), Philosophers Look at Quantum Mechanics. Dordrecht: Springer.

Cantor, G. [1955]: Contributions to the Founding of the Theory of Transfinite Numbers. New York: Dover. (The original work was published in 1915.)

Cao, T. (ed.) [1999]: Conceptual Foundations of Quantum Field Theories. Cambridge: Cambridge University Press.

Church, A. [1956]: Introduction to Mathematical Logic. Princeton, NJ: Princeton University Press.

da Costa, N.C.A., and Bueno, O. [2009]: "Non-Reflexive Logics", Revista Brasileira de Filosofia 232: 181-96.

da Costa, N.C.A., Krause, D., and Bueno, O. [2007]: "Paraconsistent Logics and Paraconsistency", in Jacquette, D. (ed.), Philosophy of Logic. Amsterdam: North-Holland, 2007, pp. 791-911.

Domenech, G., Holik, F., and Krause, D. [2008]: “Q-Spaces and the Foundations of Quantum Mechanics", Foundations of Physics 38(11): 969-994.

Falkenburg, B. [2007]: Particle Metaphysics: A Critical Account of Subatomic Reality. Dordrecht: Springer.

Frege, G. [1960]: “On Sense and Reference”, in Geach, P. and Black, M., Translations from the Philosophical Writings of Gottlob Frege. Oxford: Basil Blackwell, 1960, pp. 56-78.

Forster, T. [2014]: "Quine's New Foundations", in Zalta, E.N. (ed.), The Stanford Encyclopedia of Philosophy (Fall 2014 Edition), URL = <https://plato.stanford.edu/archives/fall2014/entries/quine-nf/>.

French, S., and Krause, D. [2006]: Identity in Physics: A Historical, Philosophical, and Formal Analysis. Oxford: Oxford University Press.

Heisenberg, W. [1998]: "The Nature of Elementary Particles", in Castellani, E. (ed.), Interpreting Bodies: Classical and Quantum Objects in Modern Physics. Princeton: Princeton University Press, pp. 211-222.

Holland, P.R. [2010]: The Quantum Theory of Motion. Cambridge: Cambridge University Press. 
Krause, D. [forthcoming]: “Does Newtonian Space Provide Identity for Quantum Systems?", forthcoming in Foundations of Science.

Krause, D. and Arenhart, J.R.B. [2018]: "Quantum Non-Individuality: Background Concepts and Possibilities", in Wuppuluri, S. and Doria, F.A. (eds.), The Map and the Territory: Exploring the Foundations of Science, Thought and Reality. Dordrecht: Springer, pp. 281306.

Krause, D. and Arenhart, J.R.B. [forthcoming]: "Presenting Non-Reflexive Quantum Mechanics: Formalism and Metaphysics", forthcoming in Cadernos de História e Filosofia da Ciência.

Lowe, E.J. [2003]: "Individuation", in Loux, M.J., and Zimmerman, D.W., (eds.), The Oxford Handbook of Metaphysics. Oxford: Oxford University Press, 2003, pp. 75-95.

Lowe, E.J. [2016]. "Non-individuals", in Guay, A. and Pradeu, T. (eds.), Individuals Across the Sciences. New York: Oxford University Press, 2016, pp. 49-60.

Maitland Wright, J. D. [1973]: "All Operators on a Hilbert Space are Bounded", Bulletin of the American Mathematical Society 79(6): 1247-51.

Maudlin, T. [2018]: "The Labyrinth of Quantum Theory https://arxiv.org/abs/1802.01834 (Accessed on June 16, 2018.)

Muller, F.A. and Saunders, S. [2008]: "Discerning Fermions", British Journal for the Philosophy of Science 59: 499-548.

Nozick, R. [1981]: Philosophical Explanations. Cambridge, MA: Harvard University Press.

Styer, D.F. et al. [2002]: "Nine Formulations of Quantum Mechanics", American Journal of Physics 70(3): 288-297.

Schrödinger, E. [1996]: Nature and the Greeks and Science and Humanism. (Foreword by Roger Penrose). Cambridge: Cambridge University Press.

Schrödinger, E. [1998]: "What is an Elementary Particle?", in Castellani, E. (ed.), Interpreting Bodies: Classical and Quantum Objects in Modern Physics. Princeton: Princeton University Press, 1998, pp. 197-210.

Szabó, Z.G. [2018]: “Compositionality”, in Zalta, E.N. (ed.), The Stanford Encyclopedia of Philosophy (Summer 2017 Edition), <https://plato.stanford.edu/archives/sum2017/entries/compositionality/>

Teller, P. [1998]: “Quantum Mechanics and Haecceities”, in Castellani, E. (ed.), Interpreting Bodies: Classical and Quantum Objects in Modern Physics. Princeton: Princeton University Press, pp. 114-141.

Weinberg, S. [1992]: Dreams of a Final Theory. New York: Vintage Books.

Weyl, H. [1950]: The Theory of Groups and Quantum Mechanics. New York: Dover.

Wilczek, F. and Devine, B. [1987]: Longing for Harmonies: Themes and Variations from Modern Physics. Penguin Books. 